\title{
Implementación del monitoreo neurofisiológico intraoperatorio en niños y adultos en el segundo y tercer nivel de atención
}

\author{
Implementation of intraoperative neurophysiologic monitoring in children and adults in \\ secondary and tertiary health care facilities
}

\begin{abstract}
Lilia Cristina De la Maza-Krzeptowsky ${ }^{1 *}$, Gabriela Romero-Esquiliano², Eduardo H. Ramírez-Segura ${ }^{3}$, Enrique De Obieta-Cruz ${ }^{4}$, Alfonso Vega-Sosa ${ }^{4}$, Alexander Cárdenas-Mejía ${ }^{5}$, Daniel San Juan-Orta ${ }^{6}$, Margot Castillo-Herrera7, Sergio J. Aguilar-Castillo ${ }^{8}$, Mario U. Ávila-OrdóñeZ ${ }^{4,9,10}$, Luz M. Cordero-Guzmán ${ }^{11}$, Rosa E. Escobar-Cedillo7, María I. Fraire-Martínez², Marisela O. Franco-Lira'13, José J. González-Jaime ${ }^{14}$, Claudia E. Paz-Navarro' ${ }^{15}$, Jaime N. Ramos-Peek ${ }^{16}$, Paul Shkurovich-Bialik ${ }^{17}$, Pedro Silva-Cerecedo ${ }^{18}$, C. Armando Tello-Valdés ${ }^{19}$, Álvaro A. Zavala-Reina ${ }^{20,21}$, Jaime López-Rodríguez ${ }^{22}$ y Ojino Sosa-García ${ }^{23}$ ${ }^{1}$ Hospital Ángeles del Pedregal, Ciudad de México; ${ }^{2}$ Universidad Autónoma Metropolitana, Ciudad de México; ${ }^{3}$ Escuela de Posgrado en Sanidad

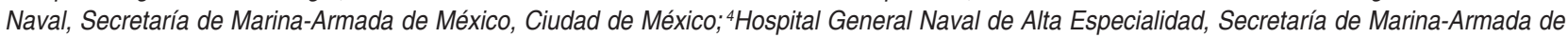
México, Ciudad de México; ${ }^{5} \mathrm{Hospital}$ General Dr. Manuel Gea González, Ciudad de México; ${ }^{\circ}$ nstituto Nacional de Neurología y Neurocirugía Manuel Velasco Suárez, Ciudad de México; ${ }^{7}$ nstituto Nacional de Rehabilitación, Ciudad de México; ${ }^{8}$ Hospital de Especialidades Centro Médico Nacional Siglo XXI, Instituto Mexicano del Seguro Social (IMSS), Ciudad de México; 'Instituto Nacional de Psiquiatría Dr. Ramón de la Fuente, Ciudad de

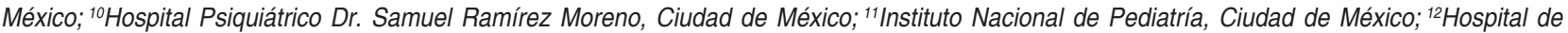
Pediatría, Centro Médico Nacional Siglo XXI, IMSS, Ciudad de México; ${ }^{13}$ Secretaría de la Defensa Nacional, Hospital Central Militar, Ciudad de México; ${ }^{14}$ Antiguo Hospital Civil de Guadalajara Fray Antonio Alcalde, Guadalajara, Jal.; ${ }^{15} \mathrm{Hospital}$ Real San José, Guadalajara, Jal.; ${ }^{16} \mathrm{Hospital}$ Ángeles Mocel, Ciudad de México; ${ }^{17}$ Centro Médico ABC, Ciudad de México; ${ }^{18} \mathrm{IMSS}$, Querétaro, Qro.; ${ }^{19} \mathrm{Hospital}$ Español, Ciudad de México; ${ }^{20} \mathrm{Hospital}$ Médica Sur, Ciudad de México; ${ }^{21}$ Hospital regional Lic. Adolfo López Mateos, Instituto de Seguridad y Servicios Sociales de los Trabajadores del Estado, Ciudad de México; ${ }^{22}$ Neurology and Neurological Sciences, and Neurosurgery Stanford University School of Medicine, CA, Estados Unidos; ${ }^{23}$ Dirección de Integración de Guías de Práctica Clínica, Centro Nacional de Excelencia Tecnológica en Salud, Ciudad de México. México
\end{abstract}

\section{Resumen}

Introducción: El monitoreo neurofisiológico intraoperatorio (MNIO) es un procedimiento que emplea técnicas neurofisiológicas con la finalidad de evaluar los sistemas motor y sensitivo durante cirugías que ponen en riesgo al sistema nervioso. Método: Se definieron el enfoque, los alcances, la población diana y las preguntas clínicas por resolver. Se realizó una búsqueda sistematizada de la evidencia por etapas. En la primera, se buscaron guías de práctica clínica; en la segunda, revisiones sistemáticas; y en la tercera, ensayos clínicos y estudios observacionales. Se utilizaron los términos MeSH y libres correspondientes, sin restricciones de lenguaje y con una temporalidad de 5 a 10 años. Se graduó la calidad de la evidencia utilizando las escalas CEPD y SIGN. Resultados: Mediante los algoritmos de búsqueda se obtuvieron 892 documentos, y se seleccionaron 58 para la inclusión de la síntesis cualitativa. Debido a la heterogeneidad entre los estudios, no fue posible realizar metaanálisis. Conclusiones: Se emitieron 18 recomendaciones, las cuales servirán como apoyo para la adecuada utilización del MNIO.

PALABRAS CLAVE: Monitoreo neurofisiológico intraoperatorio. Guía de práctica clínica. Guía.

\section{Abstract}

Introduction: Intraoperative neurophysiological monitoring (IONM) is a procedure that uses neurophysiological techniques in order to evaluate the motor and sensitive systems during surgeries that endanger the nervous system. Method: The approach,

\author{
Correspondencia: \\ *Lilia Cristina de la Maza Krzeptowsky \\ Camino a Sta. Teresa, 1055 Consultorio 920 Torre Ángeles \\ Col. Héroes de Padierna, Del. Magdalena Contreras \\ C.P. 10700 , Ciudad de México, México \\ E-mail: mtzmaza@yahoo.com.mx \\ Fecha de recepción: 20-10-2017 \\ Fecha de aceptación: 09-01-2018 \\ DOI: 10.24875/CIRU.M18000021
}

Cir Cir. 2018;86:132-139

Contents available at PubMed www.cirugiaycirujanos.com 
scope, target population, and clinical questions to be answered were defined. A systematic search of the evidence was conducted step by step; during the first stage, clinical practice guidelines were collected, during the second stage systematic reviews were obtained, and during the third stage, clinical trials and observational studies were procured. The MeSH nomenclature and free related terminology were used, with no language restrictions and a 5-10 years frame. The quality of the evidence was graded using the CEPD and SIGN scales. Results: Obtained using the search algorrhythms of 892 documents. Fifty-eight were chosen to be included in the qualitative synthesis. A meta-analysis was not possible due to the heterogeneity of the studies. Conclusions: Eighteen recommendations were issued and will support the adequate use of the IONM.

KEY WORDS: Intraoperative neurophysiological monitoring. Clinical practice. Guideline.

\section{Introducción}

El monitoreo neurofisiológico intraoperatorio (MNIO) es un procedimiento que emplea técnicas neurofisiológicas con la finalidad de evaluar los sistemas motor y sensitivo durante cirugías ortopédicas, neurológicas, vasculares y otras que ponen en riesgo al sistema nervioso'.

El uso del MNIO durante procedimientos quirúrgicos mejora el pronóstico de los pacientes sometidos a distintas cirugías. Debe ser realizado por médicos especialistas certificados por los Consejos de Neurofisiología Clínica o Medicina de Rehabilitación y que cuenten con entrenamiento en MNIO.

El MNIO se utiliza de manera sistemática en diferentes países del mundo². Los estudios publicados ofrecen evidencia para recomendar el uso del MNIO en diversos tipos de cirugías en los que existe riesgo de lesión del sistema nervioso central o periférico ${ }^{3-6}$. Está establecido que el MNIO es efectivo para predecir el incremento del riesgo de eventos adversos como paraparesia, paraplejía y cuadriplejía en cirugía espinal, mostrando una sensibilidad del $100 \%$ y una especificidad del $91 \%$ en la detección de lesión nerviosa ${ }^{4}$; para predecir la mejoría del nervio facial durante la cirugía del hemiespasmo facial ${ }^{5}$; para guiar exitosamente la resección de áreas epileptogénicas en cirugía de epilepsia6; y para alertar al cirujano de la posibilidad de lesionar nervios periféricos ${ }^{8}$.

La implementación del MNIO multimodal reduce el riesgo de complicaciones neurológicas posoperatorias en cirugías de columna en un $49.4 \%$, con un ahorro promedio de US\$ $63,387.00$ por déficit neurológico prevenido en cada paciente 9 .

En México no hay un consenso sobre el uso del MNIO en los diferentes procedimientos quirúrgicos, cuya utilización es baja, limitada o no se realiza en los centros hospitalarios públicos o privados del tercer nivel de atención. Por este motivo, surge la necesidad de desarrollar una guía de práctica clínica con el fin de dar a conocer los beneficios que este procedimiento provee a la población involucrada, ya que con su uso sistemático en las cirugías que así lo ameriten se espera una disminución en la frecuencia y la gravedad de las secuelas neurológicas posquirúrgicas.

Este artículo presenta algunas de las recomendaciones basadas en la mejor evidencia disponible con la intención de estandarizar las acciones para fundamentar que el MNIO en pacientes sometidos a cirugías que involucren estructuras raquimedulares, craneoencefálicas y de nervio periférico disminuye el riesgo de lesiones neurológicas secundarias.

\section{Recomendaciones clave}

- Los cirujanos y otros miembros del equipo quirúrgico deben ser alertados del incremento en el riesgo de efectos neurológicos adversos en pacientes con cambios transoperatorios en el MNIO.

- Se recomienda la anestesia total intravenosa con propofol durante el MNIO con potenciales evocados somatosensoriales (PESS) como la mejor opción para un óptimo registro de los potenciales motores y somatosensoriales.

- La técnica de mapeo cortical intraoperatorio en niños y adultos con o sin epilepsia usando el modelo trifásico multimodal tangencial o radial es segura y confiable para la identificación de áreas elocuentes cercanas al surco central (área somatosensorial y motora primaria).

- La electrocorticografía intraoperatoria (iECoG) es útil en cirugía de epilepsia lesional (incluyendo gangliogliomas) en niños y adultos para delimitar la zona de resección epileptogénica, guiar la resección y evaluar la resección completa.

- En las cirugías de endarterectomías carotídeas se recomienda utilizar electroencefalograma (EEG), potenciales evocados motores con estimulación eléctrica transcraneal (PEMtc) y PESS de nervio mediano y tibial para decidir en qué pacientes 
realizar puentes arteriales, iniciar las medidas de neuroprotección cerebral intraoperatorias y disminuir el riesgo de isquemia cerebral.

- El MNIO del nervio facial durante la cirugía de neuromas acústicos permite preservar la función del nervio facial en el posoperatorio inmediato y a 1 año de seguimiento.

- El MNIO de los PESS y los nervios craneales del $\mathrm{V}$ al XII, excluyendo el VIII, permite reducir los déficits neurológicos durante la cirugía de tumores de pequeño y gran tamaño de la base de cráneo.

- Se recomienda la utilización de electromiografía (EMG) continua y evocada en pacientes adultos, para la detección oportuna de lesiones neurológicas durante cirugías de raíz, plexo o nervio periférico.

\section{Método}

El grupo que desarrolló las recomendaciones se conformó con profesionales de la salud especialistas en neurofisiología clínica, medicina física y de rehabilitación, neurología, audiología, neurocirugía, cirugía plástica y reconstructiva, neuroanestesiología y anestesiología, quienes definieron el enfoque, los alcances y las preguntas clínicas. La población diana fueron niños y adultos.

Se realizó la búsqueda de guías de práctica clínica con el término MeSH "Monitoring, Intraoperative", publicadas en los últimos 5 años (de 2009 a la fecha) en idioma inglés o español. Se obtuvieron 16 resultados, de los cuales se utilizó un documento en la guía de práctica clínica en cuestión. Posteriormente se realizó la búsqueda en PubMed con los tipos de documentos de mayor evidencia clínica después de las guías de práctica clínica: revisiones sistemáticas, metaanálisis y ensayos clínicos aleatorizados; también se buscó en sitios web y en bases de datos especializadas en el área médica. Las bases consultadas fueron American University of Beirut Medical Center (un documento), PubMed Central (cuatro documentos), Springer Link (ocho documentos), OVIDSP (siete documentos), Researchgate (dos documentos), Science Direct (11 documentos) y Wiley Online Library (tres documentos).

Se graduó la calidad de la evidencia con los sistemas Clasificación de la Evidencia para la Precisión Diagnóstica (CEPD) y Scottish Intercollegiate Guidelines Network (SIGN) para emitir el nivel de evidencia (NE) y el grado de recomendación (GR). En el caso de las preguntas en las que no se encontró evidencia suficiente para realizar una recomendación se elaboró un punto de buena práctica clínica, el cual también se acordó con un grado de consenso del $100 \%$.

Se eligieron las recomendaciones clave más importantes de acuerdo con su impacto esperado. Se realizó la validación de contenido por un par clínico experto en el tema que no participó en la realización de las recomendaciones.

\section{Resultados}

Mediante los algoritmos de búsqueda se obtuvieron 776 documentos, los cuales se comprobaron por título y resumen, y se revisaron a texto completo aquellos referentes a la pregunta PICO «específica», seleccionando para la inclusión en la síntesis cualitativa 58 documentos: una guía de práctica clínica, dos revisiones sistemáticas, ocho ensayos clínicos aleatorizados, 44 estudios observacionales y un artículo no clasificado.

\section{Recomendaciones y evidencias}

\section{Cirugía de columna}

¿EI MNIO con PESS y PEMtc predice resultados adversos en cirugía de columna?

Recomendación: Los cirujanos y otros miembros del equipo quirúrgico deben ser alertados del incremento en el riesgo de efectos neurológicos adversos en pacientes con cambios transoperatorios en el MNIO (GR A).

Evidencia: En un estudio prospectivo ${ }^{6}$ se evaluó a 33 pacientes sometidos a procedimientos quirúrgicos por lesiones de la aorta torácica descendente o toracoabdominal. Se utilizó como criterio de alarma la pérdida persistente de los PESS. No se observaron cambios en 17 pacientes. Se observaron cambios en 16 , y de estos, 5 (31\%) presentaron un nuevo déficit neurológico. Prueba exacta de Fisher: $p=0.0184$.

En un estudio prospectivo ${ }^{7}$ se evaluaron 109 pacientes con MNIO sometidos a cirugía de resección de tumor espinal: 23 intramedulares, 41 intradurales-extramedulares y 45 epidurales. Durante la intervención quirúrgica se llevó a cabo la aplicación de esteroides sistémicos, hipotermia local y otras intervenciones. Se utilizaron como criterios de alarma la disminución en la amplitud de los PESS de más del $50 \%$ y el incremento de la latencia mayor del $10 \%$. 
No hubo cambio en 84 pacientes y 25 sí tuvieron cambios, de los cuales cuatro (16\%) presentaron un nuevo déficit neurológico o se agravó el preexistente (una paraplejía, una cuadriplejía y empeoramiento en dos pacientes con paraparesias preexistentes). Prueba exacta de Fisher: $p=0.00369$.

Se evaluaron 69 pacientes de manera prospectiva con PESS y PEMtc en diferentes procedimientos de cirugía de columna ${ }^{7}$, utilizando como criterio de alarma la disminución en la amplitud $>50 \%$ o el incremento en la latencia $>10 \%$. Cuarenta y nueve pacientes permanecieron sin cambios, mientras que 20 presentaron cambios, de los cuales ocho (40\%) tuvieron un nuevo déficit neurológico.

En un estudio prospectivo ${ }^{7}$ se evaluaron 52 pacientes sometidos a cirugías de columna vertebral en resección de tumor, trauma, espondilolistesis, escoliosis, malformaciones vasculares y quistes dorsales. Se realizó MNIO con PESS a 38 pacientes y con PEMtc a 12. Se empleó como criterio de alarma la disminución en la amplitud de PESS $>50 \%$ y de los PEMtc $>60 \%$. No se observaron cambios en 45 pacientes y solo cinco presentaron cambios, dos (40\%) de ellos con déficit neurológico (uno con paraplejía posterior a la cirugía y otro con paraparesia). Prueba exacta de Fisher: $p \leq 0.0158^{7}$ (NE I).

En pacientes sometidos a cirugía de columna con $M N I O$, ¿existe alguna modalidad anestésica a fin de favorecer la obtención de respuestas neurofisiológicas transoperatorias?

Recomendación: Se recomienda la anestesia total intravenosa con propofol durante el MNIO con PESS como la mejor opción para un óptimo registro de los potenciales motores y somatosensoriales (GR C).

Evidencia: En un estudio comparativo de anestesia total intravenosa con propofol versus anestesia inhalada con isoflurano en 60 pacientes sometidos a cirugía de columna con PESS, se observó que en el grupo de isoflurano la amplitud de los PESS fue significativamente $<1.5 \mathrm{mV}$, frente a $2.4 \mathrm{mV}(p=0.005)$ en el grupo de propofol ${ }^{10}$ (NE 2+).

\section{Cirugías intracraneales}

¿El uso de MNIO permite la localización de áreas elocuentes cerebrales en procedimientos neuroquirúrgicos?

Recomendación: La técnica de mapeo cortical intraoperatorio en adultos con tumores del sistema nervioso cercanos a la corteza somatosensorial primaria, usando el modelo trifásico multimodal tangencial o radial, es segura y confiable para la identificación de áreas elocuentes cercanas al surco central (área somatosensorial y motora primaria) (GR D).

Evidencia: En un estudio de una serie de 15 casos (14 adultos y 1 niño) con tumores corticales cerebrales se demostró que el modelo trifásico tangencial radial asociado con electrocorticografía y PESS, aplicando una frecuencia de $50 \mathrm{~Hz}$ y una intensidad de 3.7-12 mA (promedio de 6.2 mA), es una técnica de mapeo cortical intraoperatorio segura en pacientes despiertos o bajo sedación, porque se requieren menores intensidades de corriente eléctrica e inducen menor número de crisis epilépticas (7\%); y es confiable en la identificación de áreas elocuentes cercanas al surco central (área somatosensorial y motora primaria), lo que permite la resección de tumores en estas áreas, con unos déficits posoperatorios similares a los de otras técnicas descritas (13\%). La técnica de estimulación eléctrica directa utilizando el modelo trifásico tangencial de baja intensidad parece ser segura y reduce el riesgo de crisis intraoperatorias ${ }^{11}$ (NE 3).

¿Es posible localizar con la iECoG áreas irritativas corticales en pacientes sometidos a cirugía de epilepsia?

Recomendación: La iECoG en cirugía de epilepsia temporal es útil para caracterizar ciertos patrones electrocorticográficos que se correlacionan con subtipos histopatológicos de displasias corticales focales (DCF) y permite determinar la extensión de la resección, lo que puede influir en el resultado electroclínico posoperatorio (GR D).

Evidencia: En una serie de 22 adultos epilépticos, 16 de ellos con esclerosis hipocampal y DCF tipo 1 y seis solo con esclerosis hipocampal, sometidos a cirugía de epilepsia en quienes se realizó iECoG evaluando la frecuencia y la morfología de la actividad epiléptica, se encontró que un patrón eléctrico específico se asoció a subtipos histopatológicos de DCF (Palmini tipo 1) en pacientes con epilepsia del lóbulo temporal y patología dual'12 (NE 3).

Recomendación: La iEcoG es útil en cirugía de epilepsia lesional (incluyendo gangliogliomas) en niños y adultos para delimitar la zona de resección epileptogénica, guiar la resección y evaluar la resección completa (GR D).

Evidencia: En una serie de 157 niños y adultos epilépticos sometidos a evaluación integral perioperatoria de cirugía de epilepsia lesional utilizando IECoG en diferentes regiones cerebrales, se observó una mejoría en la iECoG posoperatoria en el $80 \%$ de los pacientes, la cual se correlacionó significativamente con una mejoría clínica, determinándose una sensibilidad del 100\%, 
una especificidad del $68.3 \%$, un valor predictivo positivo del $89.9 \%$ y un valor predictivo negativo del $100 \%$. El $73 \%$ de los pacientes tuvieron un resultado bueno de acuerdo con la escala de Engel I y II en un seguimiento promedio de 18.2 (12-94) meses ${ }^{13}$ (NE 2+).

Recomendación: La resección de cavernomas de diferentes lóbulos asociados a epilepsia en niños y adultos guiada por iECoG permite resecciones más extensas y predice un excelente control de la epilepsia a los 2 años (GR B).

Evidencia: Un estudio de casos y controles que incluyó 102 pacientes adultos, con una edad promedio de 35.4 años, con cavernomas en diferentes lóbulos y epilepsia, sometidos a cirugía guiada por iECoG para realizar lesionectomía o una resección más extensa, con un seguimiento de 37 meses, demostró independientemente de la localización del cavernoma un excelente control de la epilepsia, con un resultado Engel de clase I en el $88 \%$ de los pacientes a los 2 años. La iECoG permitió resecciones más extensas. El uso de la iECoG en los casos de cavernomas del lóbulo temporal mostró la siguiente distribución de resultados libres de crisis en pacientes sin $\mathrm{IECoG}$ vs. guiados con $\mathrm{iECoG}$ : $79 \%$ (29) vs. 91\% (23) pacientes a los 6 meses posresección, 77\% (22) vs. $90 \%$ (20) al año, y $79 \%$ (14) vs. $83 \%$ (18) a los 2 años $^{14}$ (NE 2++).

¿El uso de MNIO en endarterectomías carotídeas ayuda a identificar pacientes que requieren puente arterial o a prevenir la pérdida de funcionalidad cortical durante el procedimiento?

Recomendación: En la cirugía de endarterectomía carotídea se recomienda utilizar EEG, PEMtc y PESS de nervio mediano y tibial para decidir a qué pacientes realizar puentes arteriales, iniciar las medidas de neuroprotección cerebral intraoperatorias y disminuir el riesgo de isquemia cerebral (GR B).

Evidencia: En un estudio multicéntrico en seis centros hospitalarios en el que se realizaron 668 endarterectomías carotídeas con monitoreo mediante EEG y PESS de nervios medianos y tibiales, se determinó que un decremento $\geq 50 \%$ de la amplitud de la señal cortical, en el EEG o los PESS, indicó la necesidad de realizar un puente arterial o iniciar neuroprotección cerebral, o ambos, durante la cirugía. Los autores reportaron que en 150 de 668 cirugías se notó una disminución de la amplitud $\geq 50 \%$ en el EEG o los PESS. Posterior a la cirugía, ningún paciente presentó datos de isquemia cerebral. El puente arterial selectivo basado en el neuromonitoreo disminuyó a casi cero el riesgo de infarto cerebral cuando el MNIO era realizado por personal capacitado y con los protocolos adecuados $^{15}$ (NE 2++).

¿El MNIO del nervio facial en pacientes sometidos a cirugía del ángulo pontocerebeloso ayuda a identificar y preservar la función a corto y largo plazo?

Recomendación: El monitoreo de los PEM del nervio facial durante la cirugía descompresiva del nervio facial predice el resultado posoperatorio inmediato y a largo plazo en adultos con espasmo hemifacial (GR D).

Evidencia: Una serie de casos de 25 pacientes adultos mostró que la reducción de la amplitud de los PEM del nervio facial ocurrió después de la descompresión microvascular del espasmo hemifacial, cuyos síntomas se resolvieron en el posoperatorio, sugiriendo la normalización de la excitabilidad del nervio facial ${ }^{16}$ (NE 3).

¿El uso de MNIO en cirugías extracraneanas del nervio facial ayuda a identificar y preservar su función?

Recomendación: El monitoreo del nervio facial con PEMtc permite la preservación de la función posoperatoria del nervio facial en cirugías de tumores del ángulo pontocerebeloso sin necesidad de visualizar directamente el nervio facial (GR C).

Evidencia: Un estudio prospectivo en 68 pacientes adultos con tumores del ángulo pontocerebeloso y cinco con neuralgia del trigémino obtuvo respuestas en 57 de 68 pacientes, correlacionando positivamente la amplitud de los PEMtc del nervio facial con el grado de extensión del tumor. Las tasas de amplitud del PEMtc del nervio facial al inicio y al final de la cirugía se correlacionaron con un resultado satisfactorio temprano de la función del nervio facial, que mostró como resultados: $86 \%$ en clasificación de función del nervio facial House-Brackmann (HB) I o II, $67 \%$ en HB III, $33 \%$ en HB IV y $15 \%$ o menos en $\mathrm{HB}$ V o HB VI ${ }^{18}$ (NE 2+).

Como complemento de la EMG intraoperatoria, los PEMtc fueron superiores en dos aspectos: en primer lugar, en la identificación de lesiones latentes de los nervios faciales prequirúrgicamente, y segundo, en monitorizar la integridad del nervio sin su visualización directa. En todos los pacientes cuyas tasas de PEMtc en los músculos orbicularis oculis permanecieron $>50 \%$ de la amplitud tuvieron una función satisfactoria del nervio facial (HB I y II) en el posoperatorio inmediato y a largo plazo (24.4 meses $)^{18}$ (NE 2+).

Recomendación: La electromiografía evocada $(\geq 0.05 \mathrm{~mA}$ ) del nervio facial durante la cirugía de schwannomas vestibulares predice la parálisis facial 
permanente y mejora las tasas de preservación de la función del nervio facial en el posoperatorio (GR B).

Evidencia: Un estudio que incluyó 477 adultos con schwannomas vestibulares mostró que la electromiografía evocada ( $\geq 0.05 \mathrm{~mA}$ ) del nervio facial tiene una especificidad del $90 \%$, una sensibilidad del $29 \%$, un valor predictivo positivo del $68 \%$ y un valor predictivo negativo del $68 \%$ en la detección de parálisis facial permanente. El valor predictivo negativo disminuye significativamente con el incremento del tamaño del tumor y el riesgo de parálisis facial posoperatoria ${ }^{19}$ (NE 2++).

Recomendación: EI MNIO del nervio facial durante la cirugía de neuroma acústico permite preservar la función del nervio facial en el posoperatorio inmediato y a 1 año de seguimiento (GR D).

Evidencia: Un estudio retrospectivo de 216 pacientes con neuromas acústicos sometidos a cirugía con MNIO de nervio facial demostró que la preservación de la amplitud de los PEM del nervio facial se correlacionó significativamente con una buena función del nervio facial en el posoperatorio inmediato y al año de seguimiento. Para evitar una parálisis facial grave se encontró útil un criterio de alarma de preservación de la amplitud $>50 \%{ }^{20}$ (NE 3).

Recomendación: Durante la cirugía de parotidectomía, el MNIO del nervio facial permite la localización y la evaluación de la función continua del nervio facial, disminuyendo significativamente la parálisis temporal, la parálisis permanente y el tiempo quirúrgico (GR A).

Evidencia: Una revisión sistemática demostró que el MNIO del nervio facial disminuye significativamente la parálisis temporal y permanente del nervio facial durante la cirugía de parotidectomía. Una respuesta evocada del nervio facial $\geq 0.5 \mathrm{~mA}$ fue predictora de parálisis posoperatoria del nervio facial. El tiempo quirúrgico se ve reducido con el uso de MNIO del nervio facial ${ }^{21}$ (NE 1+).

Recomendación: EI MNIO de los PESS y de los nervios craneales V-XII, excluyendo el VIII, permite reducir los déficits neurológicos durante la cirugía de tumores de pequeño y gran tamaño de la base de cráneo (GR C).

Evidencia: El uso de MNIO de los pares craneales X, XI y XII utilizando EMG libre y respuestas motoras evocadas, en 123 adultos sometidos a cirugías de tumores de base de cráneo, ayudó a reducir la tasa de lesión de las estructuras nerviosas por la manipulación quirúrgica mediante la identificación del curso del nervio craneal en pacientes con una anatomía muy distorsionada. Estos factores pueden facilitar la resección macroscópica total del tumor con preservación de los nervios craneales ${ }^{22}$ (NE 2-).

¿El uso del MNIO durante la descompresión microvascular del VIII nervio craneal permite la preservación de la audición?

Recomendación: Se recomienda utilizar monitoreo con potenciales evocados auditivos de tallo cerebral (PEATC) en cirugías de descompresión microvascular del nervio facial para prevenir la disminución de la audición; no obstante, no se han establecido los patrones que previenen la pérdida de la audición (GR C).

Evidencia: En un estudio retrospectivo que incluyó 93 pacientes que fueron sometidos a descompresión microvascular con MNIO del VII nervio craneal por espasmo hemifacial, se utilizaron PEATC para evaluar la función del VII nervio craneal. Se empleó la clasificación de pérdida de la audición de la American Academy of Otolaryngology-Head and Neck Surgery para evaluar los resultados. El $26.88 \%$ de los pacientes tuvieron un cambio en la audición, y de estos, el $17.2 \%$ fueron clasificados en clase $B$ y el $9.68 \%$ en clase $C / D$ ( $\geq 50 \mathrm{~dB}$, audición no útil). Los cambios en las latencias interpico no incrementaron la probabilidad de pérdida de la audición ${ }^{23}$ (NE 2+).

\section{Cirugías que involucran el sistema nervioso periférico}

En los pacientes sometidos a cirugías que involucran el sistema nervioso periférico, ¿el MNIO utilizando PESS tronculares disminuye las lesiones neurológicas asociadas al procedimiento?

Recomendación: Se recomienda la utilización de PESS tronculares, en pacientes pediátricos y adultos, para la detección oportuna de lesión neurológica durante la cirugía de raíz, plexo o nervio periférico (GR D).

Evidencia: En una serie de casos de 233 pacientes pediátricos, en cirugía de alargamiento y corrección de deformidades en miembros pélvicos, se determinó la utilidad de los PESS para detectar lesión nerviosa, encontrando una sensibilidad del $100 \%$, una especificidad del $91 \%$, un valor predictivo positivo del $100 \%$ y un valor predictivo negativo del $91 \%^{4}$ (NE 3).

En los pacientes sometidos a cirugías que involucran el sistema nervioso periférico, ¿el MNIO con EMG continua y evocada disminuye las lesiones neurológicas asociadas al procedimiento?

Recomendación: Se recomienda la utilización de EMG continua y evocada en pacientes adultos para la 
detección oportuna de lesiones neurológicas durante la cirugía de raíz, plexo o nervio periférico (GR D).

Evidencia: Una serie de 127 pacientes adolescentes y adultos, sometidos a cirugía de cadera con registro de EMG continua, demostró que el $26 \%$ presentó descargas neuromiogénicas anormales indicativas de irritación nerviosa, lo que permitió cambios en la conducta quirúrgica al retirar el estímulo nocivo. Solo el $0.7 \%$ presentó déficit del nervio peroneo a más de 1 año de seguimiento ${ }^{24}$ (NE 3).

En los pacientes sometidos a cirugías que involucran el sistema nervioso periférico, ¿el MNIO con PEMtc disminuye las lesiones neurológicas asociadas al procedimiento?

Recomendación: Se recomienda la utilización de PEMtc en pacientes adultos para la detección oportuna de lesiones del plexo braquial o del nervio periférico durante la artroplastia de hombro y la fijación de fractura proximal del húmero, siendo necesario el registro simultáneo de múltiples músculos para lograr una mayor sensibilidad (GR D).

Evidencia: En una serie de 30 pacientes adultos sometidos a artroplastia de hombro con registro de PEMtc en seis músculos de la extremidad involucrada se registraron 30 alertas en 17 pacientes; el 97\% de ellas mostraron una disminución de la amplitud $>50 \%$ en Ios PEMtc, y en el $46.7 \%$ de ellas hubo evidencia de lesión en varios nervios ${ }^{25}$ (NE 3).

En los pacientes sometidos a cirugías que involucran el sistema nervioso periférico, ¿el MNIO con potenciales de acción de nervio (PAN) disminuye las lesiones neurológicas asociadas al procedimiento y ayuda a definir el procedimiento quirúrgico?

Recomendación: Se recomienda la utilización del registro de PAN en cirugía de plexo y nervio periférico para: 1) identificar el nervio; 2) localizar lesiones preexistentes en el nervio; 3) determinar la continuidad funcional a través de la lesión; 4) identificar avulsión de raíz; 5) identificar sitios para toma de biopsia; 6) prevenir el daño al nervio sano durante la cirugía; y 7) ayudar a seleccionar la técnica quirúrgica adecuada para lograr una mejor recuperación funcional (GR D).

Evidencia: En un análisis retrospectivo de 1736 pacientes con 3459 nervios lesionados, se realizó registro de los PAN a través de lesiones en continuidad para identificar el daño y definir la técnica quirúrgica ideal (neurólisis, reparación parcial, neurorrafia o reconstrucción con injerto). En los pacientes con registro de PAN y neurólisis, la recuperación funcional igual o mayor al grado 3 según la escala del Louisiana State University Health Science Center (LSUHSC) fue del $94.7 \%$. En los mixta, la recuperación funcional igual o mayor al grado 3 según la escala LSUHSC fue del $94 \%$. En los pacientes sin registro de PAN y reconstrucción con injerto, la recuperación igual o mayor al grado 3 según la escala LSUHSC fue del $56 \%{ }^{26}$ (NE 3).

\section{Conclusiones}

Este artículo presenta algunas de las recomendaciones de la guía de MNIO que fue realizada por un grupo multidisciplinario de profesionales de diferentes especialidades clínicas y quirúrgicas, permitiendo abordar el tema desde diferentes puntos de vista y enriqueciendo la búsqueda y la información final. Pretendemos con ellas homologar los criterios de la utilidad del MNIO, sistematizar su práctica y facilitar la comunicación interdisciplinaria.

EI MNIO debe ser utilizado para brindar al paciente la mayor seguridad en el tratamiento quirúrgico reduciendo los riesgos de lesión neurológica posoperatoria, y para proporcionars al médico información en tiempo real sobre la integridad funcional de las estructuras nerviosas involucradas en el procedimiento.

Necesitamos continuar reuniendo evidencias de la utilidad el MNIO para validar su importancia en nuestra población, y actualizarlas continuamente para asegurar la vigencia de las recomendaciones.

\section{Nota}

Para mayor información sobre la guía de práctica clínica Implementación del monitoreo neurofisiológico intraoperatorio en niños y adultos en el segundo y tercer niveles de atención consulte el sitio web del Catálogo Maestro de Guías de Práctica Clínica del CENETEC (http://www.cenetec-difusion.com/CMGPC/SS-721-14/ER.pdf).

\section{Responsabilidades éticas}

Protección de personas y animales. Los autores declaran que para esta investigación no se han realizado experimentos en seres humanos ni animales.

Confidencialidad de los datos. Los autores declaran que en este artículo no aparecen datos de pacientes.

Derecho a la privacidad y consentimiento informado. Los autores declaran que en este artículo no aparecen datos de pacientes.

\section{Agradecimientos}

Se agradece a las autoridades de las instituciones a las cuales pertenecen los autores. 


\section{Conflicto de intereses}

Los autores declaran que no existe conflicto de intereses.

\section{Financiamiento}

\author{
Los autores declaran que no recibieron financia- \\ miento para este trabajo.
}

\section{Bibliografía}

1. Skinner SA, Cohen BA, Morledge DE, McAuliffe JJ, Hastings JD, Yingling CD, et al. Practice guidelines for the supervising professional: intraoperative neurophysiological monitoring. J Clin Monit Comput. 2014;28:103-111.

2. Malhotra NR, Shaffrey $\mathrm{Cl}$. Intraoperative electrophysiological monitoring in spine surgery. Spine (Phila Pa 1976). 2010;35:2167-79.

3. Ney JP, van der Goes DN. Evidence-based guideline update: intraoperative spinal monitoring with somatosensory and transcranial electrical motor evoked potentials. Report of the Therapeutics and Technology Assessment Subcommittee of the American Academy of Neurology and the American Clinical Neurophysiology Society. Neurology. 2012;79:292.

4. Makarov MR, Samchukov ML, Birch JG, Cherkashin AM, Sparagana SP Delgado MR. Somatosensory evoked potential monitoring of peripheral nerves during external fixation for limb lengthening and correction of deformity in children. J Bone Joint Surg Br. 2012:94:1421-6.

5. Sekula RF Jr, Bhatia S, Frederickson AM, Jannetta PJ, Quigley MR, Small GA, et al. Utility of intraoperative electromyography in microvascular decompression for hemifacial spasm: a meta-analysis. Neurosurg Focus. 2009;27:E10.

6. Simon MV, Cole AJ, Chang EC, Buchbinder BR, Stufflebeam SM, Nozari A, et al. An intraoperative multimodal neurophysiologic approach to successful resection of precentral gyrus epileptogenic lesions. Epilepsia. 2012;53:75-9.

7. Nuwer MR, Emerson RG, Galloway G, Legatt AD, Lopez J, Minahan R, et al.; American Association of Neuromuscular and Electrodiagnostic Medicine. Evidence-based guideline update: intraoperative spinal monitoring with somatosensory and transcranial electrical motor evoked potentials. J Clin Neurophysiol. 2012;29:101-8.

8. Sutter M, Hersche O, Leunig M, Guggi T, Dvorak J, Eggspuehler A. Use of multimodal intra-operative monitoring in averting nerve injury during complex hip surgery. J Bone Joint Surg Br. 2012;94:179-84.

9. Ney JP, van der Goes DN, Watanabe JH. Cost-effectiveness of intraoperative neurophysiological monitoring for spinal surgeries: beginning steps. Clin Neurophysiol. 2012;123:1705-7.

10. Liu EH, Wong HK, Chia CP, Lim HJ, Chen ZY, Lee TL. Effects of isoflurane and propofol on cortical somatosensory evoked potentials during comparable depth of anaesthesia as guided by bispectral index. $\mathrm{Br} J$ Anaesth. 2005:94:193-7.

11. Korvenoja A, Kirveskari E, Aronen HJ, Avikainen S, Brander A, Huttunen J, et al. Sensorimotor cortex localization: comparison of magnetoencephalography, functional MR imaging, and intraoperative cortical mapping. Radiology. 2006;241:213-22.

12. Morales Chacón L, Estupiñán B, Lorigados Pedre L, Trápaga Quincoses O, García Maeso I, Sánchez A, et al. Microscopic mild focal cortical dysplasia in temporal lobe dual pathology: an electrocorticography study. Seizure. 2009;18:593-600.

13. Tripathi M, Garg A, Gaikwad S, Bal CS, Chitra S, Prasad K, et al. Intra-operative electrocorticography in lesional epilepsy. Epilepsy Res. 2010;89:133-41.

14. Van Gompel JJ, Rubio J, Cascino GD, Worrell GA, Meyer FB. Electrocorticography-guided resection of temporal cavernoma: is electrocorticography warranted and does it alter the surgical approach? J Neurosurg. 2009;110:1179-85.

15. Liu H, Di Giorgio AM, Williams ES, Evans W, Russell MJ. Protocol for electrophysiological monitoring of carotid endarterectomies. J Biomed Res. 2010;24:460-6

16. Fukuda M, Oishi M, Hiraishi T, Fujii Y. Facial nerve motor-evoked potential monitoring during microvascular decompression for hemifacial spasm. J Neurol Neurosurg Psychiatry. 2010;81:519-23.

17. Matthies C, Raslan F, Schweitzer T, Hagen R, Roosen K, Reiners K. Facial motor evoked potentials in cerebellopontine angle surgery: technique, pitfalls and predictive value. Clin Neurol Neurosurg. 2011;113:872-9.

18. Fukuda M, Oishi M, Hiraishi T, Saito A, Fujii Y. Intraoperative facial nerve motor evoked potential monitoring during skull base surgery predicts long-term facial nerve function outcomes. Neurol Res. 2011;33:578-82.

19. Sughrue ME, Kaur R, Kane AJ, Rutkowski MJ, Kaur G, Yang I, et al. The value of intraoperative facial nerve electromyography in predicting facial nerve function after vestibular schwannoma surgery. $\mathrm{J}$ Clin Neurosci. 2010;17:849-52.

20. Amano M, Kohno M, Nagata O, Taniguchi M, Sora S, Sato H. Intraoperative continuous monitoring of evoked facial nerve electromyograms in acoustic neuroma surgery. Acta Neurochir (Wien). 2011;153:1059-67.

21. Eisele DW, Wang SJ, Orloff LA. Electrophysiologic facial nerve monitoring during parotidectomy. Head Neck. 2010;32:399-405.

22. Topsakal C, Al-Mefty O, Bulsara KR, Williford VS. Intraoperative monitoring of lower cranial nerves in skull base surgery: technical report and review of 123 monitored cases. Neurosurg Rev. 2008;31:45-53.

23. Thirumala PD, Kassasm AB, Habeych M, Wichman K, Chang YF, Gardner $\mathrm{P}$, et al. Somatosensory evoked potential monitoring during endoscopic endonasal approach to skull base surgery: analysis of observed changes. Neurosurgery. 2011;69(1S):64-76.

24. Pring ME, Trousdale RT, Cabanela ME, Harper CM. Intraoperative electromyographic monitoring during periacetabular osteotomy. Clin Orthop Relat Res. 2002;(400):158-64.

25. Nagda SH, Rogers KJ, Sestokas AK, Getz CL, Ramsey ML, Glaser DL, et al. Neer Award 2005: Peripheral nerve function during shoulder arthroplasty using intraoperative nerve monitoring. J Shoulder Elbow Surg. 2007;16(3S):S2-8.

26. Robert EG, Happel LT, Kline DG. Intraoperative nerve action potential recordings: technical considerations, problems, and pitfalls. Neurosurgery. 2009;65(4S):A97-104. 